\title{
A Fundação do JEAB e o Isolamento Histórico da Análise do Comportamento ${ }^{1},{ }^{2}$ \\ ARTIGO ORIGINAL
}

Robson Nascimento da $\mathrm{Cruz}^{3}$

Pontifícia Universidade Católica de São Paulo

\begin{abstract}
RESUMO - A ocupação de lugares acadêmicos e institucionais denota a expansão da Análise do Comportamento, entre as décadas de 1940 e 1950. Todavia, é a partir desse momento que a imagem de isolamento da ciência skinneriana é difundida entre adeptos e críticos da área. Neste artigo, esse ambíguo cenário foi analisado por meio do exame histórico da fundação do primeiro periódico da Análise do Comportamento: o Journal of the Experimental Analysis of Behavior (JEAB). O argumento apresentado é de que a fundação do $J E A B$ denota episódio emblemático de um fenômeno perene na história da Análise do Comportamento, a saber, sua ininterrupta expansão institucional e científica seguida de sua crescente representação de isolamento na comunidade científica
\end{abstract}

Palavras-chave: Skinner, Burrhus Frederic, história da psicologia, análise do comportamento, JEAB

\section{The Foundation of the JEAB and the Historical Isolation of Behavior Analysis}

\begin{abstract}
The occupation of academic and institutional places denotes an expansion of behavior analysis between the 1940s and 1950s. However, the image of isolation of the Skinnerian science also started to become widespread among supporters and critics of the area during this phase. In this article, this ambiguous scenario was analyzed by examining aspects surrounding the history of the foundation of the first behavior analysis journal: Journal of the Experimental Analysis of Behavior (JEAB). The foundation of JEAB denotes an emblematic episode for understanding a perennial phenomenon in the history of behavior analysis: its continuous institutional and scientific expansion followed by its growing representation of isolation in the scientific community
\end{abstract}

Keywords: Skinner, Burrhus Frederic, history of psychology, behavior analysis, JEAB

As primeiras décadas da Análise do Comportamento denotam um fenômeno histórico paradoxal. Por um lado, seu fundador, o psicólogo experimental B. F. Skinner (19041990), obtinha crescente reconhecimento científico, gozava dos principais privilégios da elite acadêmica estadunidense e publicava suas pesquisas nos mais renomados veículos de comunicação científica (Myers, 1970; Perlman, 1980; Smith $\&$ Morris, 2004; Wright, 1970). Por outro lado, desprovida de reconhecimento, a primeira geração de adeptos da sua ciência - naquele momento, denominados condicionadores operantes - sofria seguidas dificuldades para ocupar postos acadêmicos satisfatórios e publicar seus trabalhos nos principais periódicos da psicologia experimental nos Estados Unidos (Bjork, 2006; Dews, 1987; Kelleher \& Morse, 1987; Keller, 2009; Lindsley, 1987; Skinner, 1984)33. Motivo

1 O autor agradece os comentários críticos realizados por Bruno Angelo Strapasson, Pollyanna Abreu e pelos pareceristas anônimos. Todos os possíveis erros e equívocos são de inteira responsabilidade do autor.

2 Pesquisa financiada com bolsa de pós-doutorado Fapesp, Processo 15/00514-0.

3 Endereço para correspondência: Rua Agenor Alves, 68, Nazaré, Belo Horizonte, BH, Brasi. CEP: 31.990-040.E-mail:robsoncruz78@yahoo. com.br

3 As expressões Análise do Comportamento, analistas experimentais do comportamento e analistas aplicados do comportamento surgiram entre as décadas de 1950 e 1970. No entanto, o uso padronizado de tais termos somente ocorreu a partir de meados da década de 1970 . Neste artigo, tais expressões foram mantidas devido ao seu amplo conhecimento e uso pela comunidade científica principal para tanto, à época, foi a incompatibilidade entre o delineamento de sujeito único, método utilizado por Skinner, e a adesão maciça, na psicologia experimental estadunidense, de procedimentos como o teste de hipóteses, grupos controle com elevado número de sujeitos experimentais e estatística inferencial (Capshew, 1999; Rucci \& Tweney, 1980; Todorov, 2008; Winston \& Blais, 1996).

De modo a enfrentar tal incompatibilidade e a decorrente rejeição de suas pesquisas pelo mainstream da psicologia experimental estadunidense, os primeiros praticantes da ciência skinneriana empreenderam esforços responsáveis por garantir a ocupação de lugares acadêmicos, políticos e institucionais propícios à disseminação de suas pesquisas e, por sua vez, à sobrevivência daquela incipiente comunidade científica. Alguns desses empreendimentos foram a realização da primeira conferência de análise experimental do comportamento, em 1945 (Dinsmoor, 1987); a criação de um currículo de psicologia baseado no primeiro livro de Skinner, em 1946 (Keller \& Schoenfeld, 1949); e a publicação do primeiro livro-texto de psicologia também fundamentado nos preceitos do primeiro livro de Skinner, em 1950 (Keller \& Schoenfeld, 1950). Todavia, ainda que relevantes, tais mecanismos foram insuficientes para abrandar, ao longo da década de 1950, a rejeição de pesquisas que utilizavam o delineamento experimental de sujeito único. Por isso, outras estratégias foram necessárias para enfrentar o panorama desfavorável e demarcar, de vez, os contornos da organização comunitária daquela ciência do comportamento (Kelleher \& Morse, 1987; Lindsley, 1987; Skinner, 1984). 
O objetivo deste artigo foi examinar esse controverso cenário por meio da reconstrução histórica da principal estratégia disciplinar de enfrentamento das contínuas rejeições das pesquisas dos primeiros analistas do comportamento: a fundação, em 1958, do primeiro periódico especializado da área, o Journal of the Experimental Analysis of Behavior (JEAB), e seu papel na construção da imagem de isolamento da área. Para o exame dessa história, foram abordados: (a) a criação do $J E A B$ como expressão maior do enfrentamento à rejeição do delineamento experimental de sujeito único; (b) a reprodução interna de práticas de controle coercitivo, emitidas por parte do primeiro grupo de analistas experimentais do comportamento, como produto colateral da contínua rejeição externa; e (c) os desdobramentos da tese do isolamento mútuo entre a Análise do Comportamento e o restante da psicologia.

$\mathrm{O}$ argumento defendido neste artigo é de que os eventos envoltos à constituição do $J E A B$ denotam emblematicamente um fenômeno paradoxal propagado ao longo da história da Análise do Comportamento: sua contínua expansão disciplinar e institucional, seguida de sua crescente representação de isolamento na comunidade científica. Por último, discute-se que o presente artigo, embora seja parte de tese mais ampla acerca da organização social de um grupo científico específico (Cruz, 2013), denota um estudo de caso na história da ciência, uma vez que auxilia na compreensão do fenômeno do isolamento científico e institucional de uma comunidade científica.

\section{O Surgimento do $J E A B$ e a Busca por Autonomia na Comunidade Científica}

A emergência dos primeiros meios formais de divulgação da Análise do Comportamento se deu em função das contínuas dificuldades enfrentadas pelos praticantes dessa ciência para publicar suas pesquisas em importantes veículos de comunicação científica assim como pelo sentimento compartilhado, intragrupo, de isolamento (Bjork, 2006; Skinner 1984). A fundação, em 1958, do primeiro periódico de Análise do Comportamento, o $J E A B$, teve a função de lidar com os obstáculos para divulgar a área, pois, durante toda a década de 1950, os adeptos da ciência skinneriana ainda sofriam contínuas desaprovações de suas pesquisas (Skinner, 1984). Mesmo pesquisadores vinculados diretamente a Skinner sofreram sanções por utilizar o delineamento experimental de sujeito único. Charles B. Ferster, por exemplo, o principal colaborador de Skinner na extensa série de experimentos que resultou no livro Schedules of Reinforcement (1957), teve artigos de sua autoria recusados com fortes críticas (Brady, 1987; Dews, 1987; Kelleher \& Morse, 1987).

Herrnstein (1987) descreve episódio ocorrido no começo dos anos 1950 protagonizado por Fester e simbólico dos efeitos das constantes recusas às pesquisas dos primeiros adeptos do projeto científico de Skinner e de sua função no estabelecimento do primeiro periódico da área:

Em algum ponto, me lembro de Fester entrando nervoso no escritório que eu partilhava com Morse, Blough, Anliker e Azrin, acenando uma carta de rejeição do Journal of Comparative and Physiological Psychology (JCPP), provavelmente do editor.
Eles queriam testes estatísticos. Esta indignação, primeiro, pela exigência por estatísticas inferenciais inúteis e, segundo, pela mão pesada do editor de um periódico, foi do meu ponto de vista a semente que levou à criação do $J E A B$. (p. 449)

Em conformidade com essa visão histórica, Brady (1987) recorda que, entre o final da década de 1940 e o início da década de 1950, "talvez o periódico de maior prestígio em seu tempo o - Journal of Comparative and Physiological Psychology..." (p. 458), na figura do seu editor Harlow, então presidente da APA, rejeitou com duras críticas inúmeras pesquisas que recorriam ao delineamento experimental de sujeito único. As diretrizes editoriais daquele periódico evidenciavam, no início da década de 1950, o panorama adverso. Assim, em editorial, do primeiro número daquela publicação, em 1951, promulgou-se que: "A discussão [dos artigos submetidos] deve ser limitada à interpretação e significância dos dados realmente obtidos...; dados obtidos em termos de hipóteses não testadas e não testáveis não têm lugar no JCPP...; a apresentação de dados derivados de pesquisas com sujeitos individuais deve ser evitada sempre que possível" (Harlow, 1951, p. 1). Em praticamente todos os relatos comemorativos da criação do $J E A B$, sua origem é, assim, identificada como resultado da rejeição ao delineamento experimental de sujeito único (Catania, 2008; Dews, 1987; Dinsmoor, 1987; Kelleher \& Morse, 1987; Keller, 2009, 1987; Laties, 1987; Lindsley, 1987; Skinner, 1984, 1987; Verhave, 1987). Por isso, como sugere Laties (2008, p. 96), a indignação foi o principal motivo para a fundação de um novo periódico.

Fester foi apontado pelos demais envolvidos na criação do $J E A B$ como um dos mais indignados de todos os analistas do comportamento e o responsável por levar adiante o plano de um periódico da área, já em andamento, naquele período, unindo os demais praticantes do campo em torno de uma causa comum. O planejado concretizou-se em uma reunião informal, durante o encontro da Eastern Psychological Association (EPA), de 1957, quando os analistas do comportamento ali presentes concordaram que as constantes rejeições às suas pesquisas haviam atingindo um limite insuportável (Brady, 1987; Dews, 1987; Gilbert, 1987; Herrnstein, 1987; Kelleher \& Morse, 1987; Laties, 1987; Lindsley, 1987; Schoenfeld, 1987; Skinner, 1987).

Assim, o $J E A B$ foi fundado em uma reunião, em um quarto de hotel, durante o encontro da $E P A$, em 12 de abril de 1957. O episódio denota, emblematicamente, a informalidade parcialmente imposta àquele grupo (Laties, 1987, p. 496), uma vez que, para amenizar as dificuldades de divulgação científica de suas pesquisas, era prática comum entre os primeiros analistas experimentais do comportamento a realização de sessões informais, nos quartos dos hotéis onde aconteciam os encontros da EPA, da American Psychological Association (APA) e de outras associações científicas (Kelleher \& Morse, 1987). Essas sessões serviam, ao mesmo tempo, para o compartilhamento das experiências negativas decorrentes das constantes rejeições e para o fortalecimento do sentimento intragrupo de hostilidade externa, o que intensificava o entendimento da necessidade urgente de uma alteração daquela situação precária (Laties, 1987).

A origem do $J E A B$ envolveu mais elementos do que aqueles descritos até aqui. Todavia, para os propósitos do 
presente artigo, salienta-se sua emergência como produto, principalmente, da rejeição sofrida pelos primeiros adeptos da ciência skinneriana, tendo em vista, como será exposto ainda neste trabalho, os impactos de tal desaprovação na organização do periódico e nas relações entre os diferentes praticantes da Análise do Comportamento e a comunidade externa ${ }^{1}$.

\section{Punindo quem os Punia e Punindo a si Mesmos}

Os efeitos da rejeição das pesquisas dos primeiros analistas experimentais do comportamento foram além da necessidade de estabelecimento de um espaço formal de divulgação científica e incidiram na relação desses cientistas com a comunidade externa, repercutindo, também, nos modos de relação interna daquele novo grupo científico. As primeiras implicações foram observadas quando os envolvidos na definição da política editorial do $J E A B$ discordaram sobre as diretrizes do periódico. $\mathrm{O}$ desacordo foi expresso por dois grupos: um deles recomendava o $J E A B$ como uma revista de Análise do Comportamento, que, contudo, acolheria pesquisas de outras perspectivas teórico-metodológicas; o outro grupo era defensor veemente da publicação, no periódico, apenas de pesquisas que utilizassem o delineamento experimental de sujeito único e estudassem tópicos definidos pela área (Lindsley, 1987; Schoenfeld, 1987).

A justificativa dos membros do primeiro grupo envolveu o argumento de que aceitar artigos que empregassem outros métodos e outras teorias era uma forma de disseminar a Análise do Comportamento e de inseri-la em debates da psicologia estadunidense, dos quais ela não participava efetivamente. Entretanto, para a decepção dos defensores dessa proposta, a decisão do segundo grupo prevaleceu e orientou as diretrizes da política editorial do $J E A B$, a qual decidiu por rejeitar qualquer artigo que não utilizasse método de pesquisa diferente do utilizado pela área. Para alguns membros do primeiro grupo, a decisão, vista como insensata, significou a utilização do mesmo procedimento que a comunidade externa empregava contra eles: a punição (Lindsley, 1987; Schoenfeld, 1987).

Como membro do grupo derrotado e integrante do primeiro corpo editorial do JEAB, Lindsley (1987) afirma que uma de suas memórias sobre a fundação do JEAB é "bem pesada e... um pouco triste" (p. 470). Além de recordar, pesarosamente, a oportunidade perdida de fundação de um espaço de divulgação da Análise do Comportamento para outros campos da psicologia, ele lamentou a possibilidade desperdiçada de estabelecer uma revista inovadora, com uma linha editorial totalmente diferente das demais publicações da APA, pois poderiam utilizar, pela primeira vez, os princípios da Análise do Comportamento para modelar comportamentos científicos efetivos e reforçadores, na edição científica ${ }^{2}$.

1 Para o detalhamento de informações sobre a história do $J E A B$, ver os números comemorativos de 30 e 50 anos da publicação, publicados no $J E A B, 1987$, volume 48, número 3, e $J E A B, 2008$, volume 89 , número 1 , respectivamente.

2 Sobre esse assunto, Lindsley (1987) descreve que a proposta inicial era aplicar princípios da análise experimental do comportamento ao processo de avaliação dos artigos submetidos no $J E A B$, de modo a
No entanto, infelizmente, na compreensão de Lindsley, a parcela conservadora venceu. Entre aqueles que propunham uma linha editorial ortodoxa, semelhante à que era imposta a eles, estavam Azrin, Brady, Herrnstein e, principalmente, Sidman. Por sua vez, Fester, Keller, Skinner e ele, Lindsley, foram os que assumiram uma postura liberal e defenderam uma política editorial inovadora (Lindsley, 1987).

A manifestação de Lindsley (1987) não é a única amostra da divergência interna quanto à definição da política editorial do $J E A B$. Outros membros ativos da primeira geração de analistas experimentais do comportamento expuseram sua insatisfação com os rumos da publicação, em seus primórdios, denunciando que o $J E A B$ reproduzia a mesma censura sofrida por seus fundadores. Para Schoenfeld (1987), por exemplo, a postura coercitiva impedia que o periódico recebesse contribuições de relevantes pesquisadores que não utilizavam, exclusivamente, a linguagem skinneriana. Ainda em suas palavras também pesarosas isso significou que:

Infelizmente, alguns de nossos editores logo adotaram a mesma rigidez e atitudes de censura que eles tinham criticado em outros periódicos, por exemplo, com respeito a um vocabulário específico que eles insistiam que um autor empregasse. Esta exclusividade e interferência inoportuna rapidamente distanciaram potenciais contribuintes, alguns dos quais eu tinha classificado como pesquisadores realmente talentosos... Para mim isto significou que se deve também considerar, quando um periódico é fundado, a modelagem do comportamento do editor. (Schoenfeld, 1987, p. 466)

Além de expor a forma como o corpo editorial do JEAB reproduzia práticas restritivas impostas aos seus fundadores, Lindsley (1987) destaca que, no início da década de 1960, o conselho editorial estendeu as mesmas práticas de controle da produção aos primeiros analistas aplicados do comportamento, justificando-se a rejeição interna pela incapacidade de as pesquisas aplicadas obterem controle experimental preciso das variáveis. A respeito disso, Lindsley apresenta, como exemplo, a rejeição, com intensas críticas, de um artigo de sua autoria que investigava o comportamento psicótico. Insatisfeito por ter um trabalho reprovado na revista que ajudou a fundar, Lindsley submeteu o artigo a outro periódico, que o aceitou sem nenhuma solicitação de alteração. "A reimpressão desse artigo venceu o prêmio anual da Associação Norte-Americana de Psiquiatria em 1962. Obviamente, os conservadores haviam vencido" (Lindsley, 1987, p. 470).

A rejeição de pesquisas aplicadas, como a de Lindsey (1987), constitui parte das justificativas para o lançamento, em 1968, do segundo periódico da área, o Journal of Applied Behavior Analysis (JABA). Como sugere Krantz (1971), a reprovação, pelo $J E A B$, de relatos de pesquisas de analistas aplicados do comportamento aconteceu em razão da concepção restrita de controle experimental utilizada pelo periódico, a qual impedia que pesquisas aplicadas alcançassem os critérios estabelecidos para publicação. Nessa perspectiva, a fundação do $J A B A$ não foi - como poderia ser presumido - uma extensão direta e necessária da produção científica veiculada no $J E A B$. Ou seja, embora utilizasse resultados de pesquisas publicadas naquele periódico, o $J A B A$

incentivar o debate e amenizar a aversão do processo de avaliação. 
não representou, precisamente, uma transição harmônica da evolução da pesquisa básica à aplicação, como se poderia entender de uma ciência definida pela relação entre três eixos de investigação: um experimental, um aplicado e um conceitual (para uma análise dessa definição, ver Carvalho Neto, 2002, e Tourinho, 1999).

Assim, a fundação do $J A B A$, diferentemente de inaugurar o início do diálogo entre dois campos do conhecimento, derivados da mesma ciência, demarcou o início do evidente distanciamento entre analistas aplicados e analistas experimentais do comportamento. O fenômeno foi, primeiramente, destacado pelos editores do JEAB, que alegaram não possuir conhecimento suficiente para avaliar as pesquisas aplicadas publicadas no $J A B A$ (Krantz, 1971). Outra prova desse distanciamento encontra-se no desconhecimento que editores e autores do $J E A B$ tinham acerca de quem eram os editores e membros do corpo editorial do $J A B A$. Krantz (1972) explica essa desinformação, entre os editores, como resultado parcial da ausência de conexão entre os problemas da pesquisa aplicada e os problemas da pesquisa básica. Sugere ainda que esse problema deriva, em alguma medida, do fato de que líderes da pesquisa aplicada em análise do comportamento, como Bijou e Baer, "não tinham uma interconexão baseada em treinamento e canais formais e informais de comunicação com as pessoas na área de pesquisa animal operante" (p. 94).

Tendo em vista a frágil conexão entre analistas experimentais e analistas aplicados, suas relações nas décadas seguintes, se não sinalizam uma explícita oposição hostil intragrupo, ao menos representam os contínuos problemas de comunicação entre os diferentes praticantes da Análise do Comportamento. Vide os editoriais do $J E A B$, do $J A B A$ e do The Behavior Analyst (BAT), que atestam um cenário de contínuas críticas mútuas entre analistas aplicados e analistas experimentais, ao longo das décadas de 1960 e 1970 (e.g., Baer, 1980; Dinsmoor, 1979; Michael, 1979; Peterson, 1978, 1979). Fenômeno verificado também ao longo das décadas de 1980, 1990 e 2000, em análises que atestam a contínua tendência ao isolamento intra-comunidade analítico comportamental (e.g., Eliott, 2005; Poling, Picker, Grossett, Hall-Johnson, \& Holbrook, 1981).

A respeito dos modos de controle da comunicação interna da Análise do Comportamento, ainda vale mencionar que pesquisadores que utilizaram a Análise do Comportamento, no final da década de 1960 , para investigar temáticas além daquelas definidas pelo $J E A B$ e $J A B A$, alegaram ter sido vítimas da intolerância interna em relação a inovações. Sobre isso, um entrevistado de Krantz (1972) alegou, “o JEAB está nos tratando como o JCPP os tratou” (p. 95). Por essa razão, para o entrevistado, as políticas editoriais do $J E A B$ e do $J A B A$ denotavam um conservadorismo inerente à área.

\section{A Tese do Isolamento Mútuo}

A contínua ampliação do número de assinaturas do $J E A B$ e do $J A B A$ bem como o volume crescente de autores nessas publicações corroboram as alegações de intensa expansão da Análise do Comportamento como comunidade científica, entre as décadas de 1960 e 1970 (Laties, 2008).
A fundação, em 1964, da divisão 25 da APA, Divisão de Análise Experimental do Comportamento - única sessão da associação científica vinculada a apenas uma perspectiva psicológica originada do trabalho de um único cientista, Skinner - e o sucessivo crescimento do número de afiliados, nas décadas de 1960 e 1970, são outras evidências do desenvolvimento da Análise do Comportamento (Guttman, 1977). Constituem, também, sinais de tal ampliação institucional e disciplinar da área a publicação de livros didáticos e manuais de aplicação e métodos de pesquisa operante, como os livros de Werner K. Honig, Operant Behavior: Areas of Research and Application (1966), e de Murray Sidman, Tatics of Scientific Research (1960).

A notável expansão da Análise do Comportamento e suas peculiaridades teóricas, metodológicas e seus modos de relação com a comunidade externa chamaram a atenção da comunidade científica, não tardando a ser foco de comentários e de estudos metacientíficos. A despeito dos diferentes enfoques, essas análises expressam o argumento de que a Análise do Comportamento era um caso exemplar do isolamento mútuo entre uma perspectiva científica e o restante do campo do qual ela fazia parte. Prenúncios da ocorrência desse fenômeno foram apregoados, ainda no final dos anos 1940, por Wendt (1949), quando declarou que o novo currículo de psicologia da Universidade Columbia, proposto por Keller e Schoenfeld (1949) e fundamentado no projeto científico skinneriano, induziria à formação de um culto científico. Para Wendt, o resultado daquele modelo de ensino seria o isolamento de seus membros em relação às outras correntes teóricas da psicologia.

Quase 20 anos após o artigo de Wendt (1949), Hearst (1967) argumenta que o previsto isolamento dos praticantes da ciência skinneriana havia se concretizado na visão de grande parte dos psicólogos estadunidenses. Ao resenhar o livro Operant Behavior: Areas of Research and Application (Honig, 1966), em um texto intitulado de "The Behavior of Skinnerians", Hearst sugere que os capítulos daquele livro designavam o isolamento da área em termos de tópicos de estudo e, principalmente, de método. Contudo, haveria, para Hearst, sinais de contribuição daquela ciência para outros psicólogos, fora do campo operante. Os capítulos sobre psicofarmacologia, desenvolvimento infantil e alterações de estados fisiológicos apontavam, segundo ele, possíveis contribuições da análise experimental do comportamento para a psicologia como um todo. Todavia, a linguagem restrita, o foco excessivo na análise de esquemas de reforçamento e o desgosto declarado, por parte dos praticantes da Análise do Comportamento, pelo mainstream da psicologia experimental já haviam instaurado, naquele contexto e momento, o que Hearst considerava uma caricatura parcialmente verdadeira daqueles que se declaravam skinnerianos. Essa caricatura parcial foi assim descrita por ele:

Para muitos outsiders, um condicionador operante é um experimentalista fechado, que passa horas infindas na análise entusiasmada dos dados cumulativos de um ou dois sujeitos, ataca qualquer coisa que pareça meramente teórica ou fisiológica, ridiculariza qualquer pessoa que já tenha usado estatísticas... ignora o trabalho de qualquer psicólogo que não publica no Journal of the Experimental Analysis of Behavior. Desde a época de J. B. Watson, nenhum grupo behaviorista parece tão certo 
do que gosta ou não gosta e tão convencido que suas técnicas e abordagem experimental não só mudarão a psicologia, mas remodelarão o mundo. (Hearst, 1967, p. 402)

Não obstante as alegações de isolamento e ortodoxia da Análise do Comportamento tenham sido identificadas desde o final da década de 1940, foram os estudos de Krantz (1971, 1972) que sistematizaram, em termos quantitativos (bibliometria) e qualitativos (entrevistas), a tese propagada, até o presente, do isolamento mútuo entre a psicologia operante e a psicologia não operante. Nesses dois estudos, Krantz verificou o padrão quantitativo de citações e autocitações no $J E A B$ e em outros periódicos de psicologia experimental, entre 1958 e 1969, e constatou que os periódicos que citavam o $J E A B$, além de o fazerem menos frequentemente do que em relação a qualquer outra publicação, apresentaram diminuição das referências ao periódico nesse intervalo. Igualmente, as mesmas publicações apresentavam elevada taxa de citações em outras publicações e, em comparação com outros periódicos investigados, o $J E A B$ expôs a maior frequência de autocitações, com manifesta tendência à sua contínua elevação.

Já em sua análise qualitativa, Krantz (1971, 1972) constatou que psicólogos adeptos de outras abordagens psicológicas julgavam que os analistas experimentais do comportamento falavam para si mesmos, garantindo apoio e reconhecimento mútuo entre os pares e se blindando, assim, das críticas externas. Porém, as entrevistas de Krantz (1972), realizadas com praticantes da Análise do Comportamento e psicólogos de outras abordagens, indicaram que o isolamento da Análise do Comportamento não significou que o recurso a conceitos e, principalmente, a métodos da abordagem se manteve dentro dos limites do grupo de praticantes daquela ciência. Alguns entrevistados asseveraram utilizar-se dos métodos operantes sem se submeter ao que intitulavam de religião skinneriana; ou seja, "muitos outsiders agiam como insiders, particularmente em seus usos da tecnologia operante, enquanto ao mesmo tempo eram críticos do condicionamento operante" (Krantz, 1972, p. 95).

Portanto, os empregos da tecnologia operante, embora apresentando coesão interna e identificação com um grupo específico, tornaram as fronteiras da Análise do Comportamento menos impermeáveis. Krantz (1972) enfatiza que, ainda assim, a identidade, especialmente dos analistas experimentais do comportamento, permaneceu distinta e não foi incorporada amplamente à psicologia, pois, mesmo em diferentes níveis, eles trabalhavam exclusivamente com base nos pressupostos do sistema científico skinneriano, o qual, conquanto provendo tecnologias utilizadas por praticantes de outras perspectivas, continuava sendo alvo de críticas e incompatível com o mainstream da psicologia experimental, no que se referia a seu método de pesquisa. Isso manteve intocável o núcleo da identidade daqueles cientistas, que possuíam plena consciência de que a rejeição externa os definia como grupo. Portanto, na amostra de Krantz, a percepção de afiliação exclusiva à ciência skinneriana foi compartilhada, principalmente, por aqueles que a praticavam antes de 1958, os quais viveram a forte experiência da hostilidade externa, a contínua rejeição de suas pesquisas, o esforço grupal para fundar o JEAB e a dedicação à criação de uma educação disciplinar em Columbia.
As pesquisas de Krantz $(1971,1972)$ forneceram o primeiro aporte empírico para corroborar a percepção histórica, existente desde o final da década de 1940, da ortodoxia e do isolamento da Análise do Comportamento. Por isso, desde então, têm servido como base comparativa para as demais pesquisas preocupadas em investigar o grau de comunicação interna e externa da área. Todavia, apesar de seu valor histórico, autores como Coleman e Mehlman (1992) alegam que a tese de Krantz produziu um efeito negativo na interpretação histórica da Análise do Comportamento. Mais precisamente, eles sugerem que o trabalho de Krantz acentuou a percepção de isolamento da Análise do Comportamento, uma vez que serviu de mote para reafirmar a posição desfavorável daquela ciência por seus críticos (e.g., Guttman, 1977).

Wyatt, Hawkins e Davis (1986) foram os primeiros a tentar refutar a tese do isolamento. Segundo eles, os usos dos achados de Krantz eram anacrônicos para interpretar a situação da Análise do Comportamento no começo da década de 1980. Razão para tanto seria o fato de que, desde a publicação dos artigos de Krantz, no início da década de 1970, ocorreu contínuo crescimento, em quantidades absolutas, de atividades acadêmicas e disciplinares da área, tais como a criação de publicações especializadas, de cursos, de linhas de pesquisas e de sociedades científicas. Para Coleman e Mehlman (1992), os resultados do estudo de Wyatt et al. (1986) provavam que a situação de isolamento da Análise do Comportamento era menos precária do que o anunciado por seus críticos. Todavia, ao replicarem parcialmente, no início da década de 1990, as análises bibliométricas de Krantz (1971,1972), concluíram que, a despeito da diminuição da taxa de autocitação no $J E A B$, esta se mantinha elevada em comparação com os demais periódicos por eles avaliados. Por isso, alertaram que "os leitores que foram perturbados pelas descobertas de Krantz deveriam, apesar das tendências favoráveis que foram descritas, estar perturbados pelos resultados do nosso estudo" (Coleman \& Mehlman, 1992, p. 48).

Ainda assim, Coleman e Mehlman (1992) argumentam que, nos estudos de Krantz (1971, 1972), o termo isolamento foi utilizado de forma limitada, pois teve como respaldo o baixo volume de comunicação, além da especialidade. Consequentemente, apenas o critério bibliométrico, utilizado nas pesquisas de Krantz, seria insuficiente para determinar uma interpretação patologizante do isolamento da Análise do Comportamento, uma vez que, se outros indicadores fossem empregados - por exemplo, o contínuo desenvolvimento disciplinar e institucional da área -, os dados de Krantz seriam relativizados. Ainda que relevante, essa alegação de Coleman e Mehlman desconsidera que dados relativos à expansão disciplinar e institucional da Análise do Comportamento não seriam indicativos necessários de maior comunicação da área para além de suas fronteiras e, até mesmo, dentro de suas fronteiras. Como sugeriu Krantz, um dos elementos que chama a atenção no isolamento da Análise do Comportamento, já nas décadas de 1950 e 1960, é que ele ocorreu à medida que a área se expandia em termos disciplinares e institucionais.

A despeito disso, Coleman e Mehlman (1992) sugerem que, talvez, o isolamento da Análise do Comportamento 
resulte de efeitos positivos do desenvolvimento da área, como sua autossuficiência intradisciplinar, seu consenso interno e a convergência teórica e metodológica entre seus praticantes. Essa afirmação foi justificada com base na alegação de que elevadas taxas de autocitação existem em especialidades de outras ciências experimentais. Embora coerente, tal comparação parece desconsiderar que ciências como a física, a química e a biologia, ainda que apresentem controvérsias internas (Latour, 1998), compartilham, de modo geral, acordos teóricos válidos para todas as suas subespecialidades. Com isso, nessas ciências, a limitação da disseminação de seus produtos intelectuais, como ocorreu na Análise do Comportamento, não é sinal necessário de isolamento. Nesse ponto, vale retomar a afirmação de Krantz (1972) de que embora as demais perspectivas em psicologia experimental estadunidenses mantivessem, também, suas especificidades e fronteiras delimitadas, apresentavam indícios mais claros de produções científicas intercambiáveis e julgavam tal interação necessária.

Vale salientar que a Análise do Comportamento não deve ser considerada a única comunidade científica a apresentar isolamento científico. Porém, no contexto histórico da psicologia experimental estadunidense das décadas de 1950 e 1960, o seu isolamento adquiriu formas peculiares, sendo um isolamento anômalo na visão, principalmente, de não praticantes da área. Vide o fato significativo de que, embora nunca tenha mantido relações amistosas e próximas com a APA (Woodward, 1996), a Análise do Comportamento compunha a única divisão dessa associação científica, no começo da década de 1960, identificada com apenas uma teoria do comportamento, de um único autor (Bjork, 2006). Isso constitui algo bem diverso do que ocorria com as demais divisões da APA, as quais, embora pudessem ter suas fronteiras demarcadas, não se identificavam como representantes de somente uma perspectiva científica, de um único psicólogo. Tais divisões apresentavam identificação com áreas da psicologia, como a psicologia educacional, a psicologia no serviço público e a psicologia clínica, entre outras que contavam com a participação de psicólogos e psicólogas de diferentes orientações teóricas e metodológicas, o que propiciava espaço de contato, por mínimo que fosse, entre seus participantes.

Não por acaso, a própria temática do isolamento tornou-se parte da interpretação histórica da Análise do Comportamento. Em extensa análise recente dos usos da tecnologia operante e da organização da Análise do Comportamento, a historiadora da psicologia Alexandra Rutherford (2009) pontuou que a estrutura comunitária da Análise do Comportamento, entre as décadas de 1950 e 1960 , determinou a coesão interna dessa ciência e foi responsável pela ampla inserção da tecnologia operante em vários âmbitos da sociedade estadunidense. No entanto, a autora considera que a mesma estrutura social foi responsável por, ao longo do tempo, tornar as inovações da tecnologia operante restritas aos praticantes da Análise do Comportamento. Em razão disso, Rutherford supõe que os resultados de Krantz (1972) são úteis para a interpretação da situação contemporânea da Análise do Comportamento como grupo científico. Assim, conclui que a tese do isolamento faz tanto sentido atualmente quanto na década de 1970, pois a Análise do Comportamento estaria ainda isolada do mainstream da psicologia, uma vez que, no presente: "Skinnerianos publicam em seus periódicos, participam de suas próprias conferências, e treinam seus estudantes em certos redutos remanescentes da Análise do Comportamento..." (Rutherford, 2009, p. 151).

Além disso, para Rutherford (2009), analistas experimentais e aplicados do comportamento mantêm uma linguagem especializada "que, não só os separou dos outros psicólogos, como criou um senso de coesão intragrupo que é palpável em qualquer encontro onde os skinnerianos predominam" (p. 151). De acordo com essa autora, entre as consequências negativas desse modo de funcionamento social estaria o fato de que tecnologias derivadas da psicologia operante - anteriormente absorvidas por outras áreas e profissionais - deixaram, ao longo do tempo, de ter suas origens identificadas naquela ciência, de modo que, atualmente, as novas descobertas da Análise do Comportamento não são consideradas de modo relevante além da própria área.

Ainda mais sintomático, na tese do isolamento, é a manutenção do tema entre seus praticantes, na atualidade. Vide os números especiais dos periódicos The Behavior Analyst e European Journal of Behavior Analysis, ambos de 2014, cuja temática central foram os problemas de comunicação da Análise do Comportamento. Embora uma síntese desse debate ultrapasse nosso propósito, o que se observa nele é a reprodução de controvérsias similares àquelas identificadas nos momentos que antecederam e sucederam a fundação do JEAB, nas décadas de 1940 e 1950. Entre elas, observa-se a persistente polêmica com respeito ao controle das balizas linguísticas da Análise do Comportamento. Algo percebido na apologia, de um lado, de uma maior permissividade e disposição para o diálogo horizontalizado com a psicologia e outras áreas do conhecimento (e.g, Schlinger, 2014) e, por outro, na extremada defesa pela separação da Análise do Comportamento do restante da psicologia (e.g., Ulman, 2014) e a necessidade de controle rígido dos usos da sua linguagem, tanto na comunidade científica quanto na arena pública (e.g., Reed, 2014).

\section{Considerações Finais}

A história de fundação do $J E A B$ unifica uma diversidade de elementos históricos da organização social da Análise do Comportamento que permitem argumentar que, à medida que tal ciência se expandiu em termos disciplinares, ela foi situada, por seus praticantes e não praticantes, como cada vez mais isolada do restante da psicologia. Pode-se dizer, contudo, que o isolamento científico não seria um evento restrito à Análise do Comportamento. A psicologia, como ciência e disciplina, tem sido caracterizada, historicamente, pela fragmentação teórica e inexistência de acordos epistemológicos e metodológicos - mínimos - entre suas diferentes abordagens (Ferreira, 2006). Ainda assim, a despeito dessa constatação, neste artigo, foi evidenciado que o isolamento da Análise do Comportamento apresentaria peculiaridades históricas marcantes, que tornaram acentuada a percepção de seu isolamento no âmbito da psicologia. 
Esse isolamento primeiro se deu em função da incompatibilidade metodológica com o mainstream da psicologia experimental estadunidense e pela adesão restrita a uma teoria, o que levou a Análise do Comportamento, diferente das demais tendências em psicologia experimental nos EUA de meados do século XX, a ser percebida como uma área e, ao mesmo tempo, como uma abordagem psicológica. Vide sua configuração, como mostramos, na APA, como a única divisão filiada a apenas uma abordagem psicológica, derivada de uma única ciência do comportamento e do trabalho de um único psicólogo. Como resultado dessa configuração, a área foi e tem sido representada como insensível e resistente para lidar com temáticas que ultrapassam as fronteiras linguísticas do próprio campo.

Ainda de especial interesse histórico e representativo de singularidades do isolamento da Análise do Comportamento foi o papel desempenhado pela punição na origem do $J E A B$ e seus efeitos na organização comunitária da área. Sobre isso, foi indicado como os debates internos que permearam a organização e a definição da política editorial do JEAB sinalizam o papel central das incessantes rejeições na organização comunitária daquele grupo. A recusa da produção científica de outras abordagens psicológicas e dos próprios analistas do comportamento é prova cabal da reverberação dos efeitos da hostilidade externa. Assim, as críticas já existentes, no final da década de 1940, de que a Análise do Comportamento constituía um grupo científico isolado e ortodoxo se ampliaram após a fundação do $J E A B$. Krantz $(1971,1972)$ corroborou tal visão histórica ao defender a tese do isolamento mútuo entre a psicologia operante e a psicologia não operante. Mesmo questionada, o que chama atenção é que tal tese mantém seu valor heurístico ainda no presente, uma vez que estudos desenvolvidos 20 e 40 anos mais tarde, inclusive para tentar mostrar seu provável anacronismo, reforçam indícios da manutenção do isolamento da Análise do Comportamento (Coleman \& Mehlman, 1992; Rutherford, 2009).

Tal compreensão dos efeitos da punição é compatível com as análises sociológicas acerca da marginalização de grupos pelo establishment. Bourdieu (1983), por exemplo, ao transpor sua noção de campo para a ciência, afirma que, assim como outros grupos sociais quando ocupam postos de autoridade, os grupos científicos à margem do establishment científico tendem a consagrar estratégias de conservação. Desse modo, o grupo científico excluído tende a manter e perpetuar uma ordem científica com a qual compactuam apenas seus praticantes. Postura assumida por parte dos fundadores do $J E A B$, já no momento de sua criação. Assim, o JEAB configurou uma ambígua estratégia de institucionalização da Análise do Comportamento, visto que, ao mesmo tempo em que foi fundado para enfrentar a rejeição e isolamento, estabeleceu, a contragosto de parte daquela comunidade científica, uma política editorial que reproduzia aquilo que buscava enfrentar: a punição resultante das rejeições de suas pesquisas.

Ainda é digno de nota observar que a temática do isolamento extrapola o cenário estadunidense. No Brasil, por exemplo, analistas do comportamento igualmente têm sugerido a existência do fenômeno do isolamento da área. $\mathrm{E}$, entre as razões para tanto, estaria, do mesmo modo que nos Estados Unidos, a manutenção por seus membros de práticas sociais coercitivas com respeito aos modos de comunicação de seus praticantes (Banaco, 1997; Rodrigues, 2002) $)^{3}$. Embora os motivos do possível isolamento da Análise do Comportamento no Brasil tenham origens diferentes daquelas encontradas nos Estados Unidos, a temática do isolamento , portanto, ultrapassa o contexto estadunidense e possivelmente é parte constituinte de sua organização, independente do contexto geográfico. Nessa perspectiva, é possível afirmar que a questão do isolamento é um tema perene na história da Análise do Comportamento, sendo parte constituinte da própria identidade dessa comunidade científica ${ }^{4}$.

Finalmente, espera-se que, tanto para os praticantes da Análise do Comportamento quanto para não praticantes, o presente artigo ultrapasse o valor de mera curiosidade histórica da ciência. Para os primeiros, entende-se que este estudo pode suscitar debates acerca da estrutura organizacional da comunidade científica da qual fazem parte e de seus prováveis desdobramentos históricos no presente. Para os não praticantes (e ainda igualmente para os praticantes), presume-se que, embora a história abordada se refira a um grupo específico, em um contexto e momento histórico particular, serve de base para o debate de aspectos microssociais inerentes ao funcionamento da ciência em diferentes campos do saber.

\section{Referências}

Andery, M. A. P. (2012). Entrevista: Maria Amália Pie Abib Andery. Boletim Paradigma, 7, 21-27.

Baer, D. M. (1980). A flight of behavior analysis. The Behavior Analyst, 4, 85-91.

Banaco, R. A. (1997). Podemos nos beneficiar das descobertas da ciência do comportamento?. Em R. A. Banaco (Ed.), Sobre comportamento e cognição: Aspectos teóricos, metodológicos e de formação em Análise do Comportamento e terapia cognitiva (pp. 543-555). Santo André, SP, Brazil: ARBytes.

Bjork, D. W. (2006). B. F. Skinner: A life. New York: Sheridan Books.

Bourdieu, P. (1983). O campo científico. Em R. Ortiz (Ed.), Pierre Bourdieu: Sociologia (pp. 122-155). São Paulo: Ática.

Brady, J. V. (1987). Back to baseline. Journal of the Experimental Analysis of Behavior, 48, 458-459.

3 O isolamento da Análise do Comportamento no Brasil necessita de maiores investigações, uma vez que a rejeição da área no Brasil estaria relacionada a questões diferentes daquelas existentes nos Estados Unidos. No Brasil, as especificidades da história da Análise do Comportamento não permitem julgá-la como um perspectiva psicológica rejeitada, quando da sua institucionalização (Matos, 1996). Razões de ordem política e ideológica precisam também ser incluídas para explicar os motivos da resistência histórica à Análise do Comportamento no país (Cruz, 2010; Rodrigues, 2002).

4 Andery (2012), outra analista do comportamento brasileira, embora não se limite a avaliar a situação da Análise do Comportamento no Brasil, igualmente alega estar a área em crescente processo de isolamento científico. Do mesmo modo, Todorov (2013) indica a manutenção histórica do problema do isolamento da Análise do Comportamento. 
Capshew, J. H. (1999). Psychologists on the march: Science, practice, and professional identity in America, 1929-1969. Cambridge, MA: Cambridge University.

Carvalho Neto, M. B. (2002). Análise do comportamento: Behaviorismo radical, análise experimental do comportamento e análise aplicada do comportamento. Interação, 6, 13-18. doi: http://dx.doi.org/10.5380/psi.v6i1.3188

Catania, C. A. (2008). The Journal of the Experimental Analysis of Behavior at zero, fifty and one hundred. Journal of the Experimental Analysis of Behavior, 89, 111-118. doi: https:// dx.doi.org/10.1901\%2Fjeab.2008.89-111

Coate, W. B. (1956). Weakening of conditioned bar-pressing by prior extinction of its subsequent discriminated operant. Journal of Comparative and Physiological Psychology, 49, 135-138.

Coleman, S. R., \& Mehlman, S. E. (1992). An empirical update (1969-1989) of D. L. Krantz's thesis that the experimental analysis of behavior is isolated. The Behavior Analyst, 15, 43-49.

Crane, D. (1972). Invisible colleges. Chicago: University of Chicago Press.

Cruz, R. N. (2013). B.F. Skinner e a Vida Cientifica: Uma história da organização social da Análise do Comportamento (Tese de doutoramento não publicada). Faculdade de Filosofia e Ciências Humanas, Universidade Federal de Minas Gerais, Belo Horizonte, MG.

Cruz, R. N. (2010). Possíveis relações entre o contexto histórico e a recepção do behaviorismo radical. Psicologia: Ciência e Profissão, 30, 478-491.

Dews, P. B. (1987). An outsider on the inside. Journal of the Experimental Analysis of Behavior, 48, 459-462.

Dinsmoor, J. A. (1951). The effect of periodic reinforcement of barpressing in the presence of a discriminative stimulus. Journal of Comparative and Physiological Psychology, 44, 354-361.

Dinsmoor, J. A. (1952). Resistance to extinction following periodic reinforcement in the presence of a discriminative stimulus. Journal of Comparative and Physiological Psychology, 45, 31-35.

Dinsmoor, J. A. (1979). A note on the historical record: MPA and MABA. The Behavior Analyst, 2, 22-24.

Dinsmoor, J. A. (1987). A visit to Bloomington: The first conference on the experimental analysis of behavior. Journal of the Experimental Analysis of Behavior, 48, 441-445.

Eliott, A. J. (2005). Self and cross-citations in the journal of applied behavior analysis and the Journal of the Experimental Analysis of Behavior: 1993-2003. Journal of Applied Behavior Analysis, 38, 559-563.

Ferreira, A. A. L. (2006). A psicologia como saber mestiço: $\mathrm{O}$ cruzamento múltiplo entre práticas sociais e conceitos científicos. História, Ciência, Saúde-Manguinhos, 13, 227-238. doi: http://dx.doi.org/10.1590/S0104-59702006000200002.

Gilbert, M. B. (1987). Memories of JEAB's mother. Journal of the Experimental Analysis of Behavior, 48, 475-477.

Guttman, N. (1977). On Skinner and Hull: A reminiscence and projection. American Psychologist, 32, 321-328.

Harlow, H. F. (1951). Publication policy for the Journal of Comparative and Physiological Psychology. Journal of Comparative and Physiological Psychology, 44, 1-2.

Hearst, E. (1967). The behavior of Skinnerians. Contemporary Psychology, 12, 402-404.
Herrnstein, R. J. (1987). Reminiscences already? Journal of the Experimental Analysis of Behavior, 48, 448-453.

Honig, W. K. (1966). Operant behavior: Areas of research an application. New York: Appleton-Century-Crofts.

Jenkins, W. O., McFann, H., \& Clayton, F. L. (1950). A methodological study of extinction following a periodic and continuous reinforcement. Journal of Comparative and Physiological Psychology, 43, 155-167.

Kelleher, R. T., \& Morse, W. H. (1987). The Yerkes connection. Journal of the Experimental Analysis of Behavior, 48, 456-457.

Keller, F. S. (2009). At my own pace: The autobiography of Fred S. Keller. New York: Sloan Publishing.

Keller, F. S., \& Schoenfeld, W. N. (1949). The psychology curriculum at Columbia College. American Psychologist, 4, 165-172.

Keller, F. S., \& Schoenfeld, W. N. (1950). Principles of psychology: A systematic text in the science of behavior. New York: Appleton-Century-Crofts.

Keller, F. S. (1987). Columbia gems. Journal of the Experimental Analysis of Behavior, 48(3), 453-455.

Krantz, D. L. (1971). The separate worlds of operant and nonoperant psychology. Journal of Applied Behavioral Analysis, 4, 61-70.

Krantz, D. L. (1972). Schools and systems: The mutual isolation of operant and non-operant psychology as a case study. Journal of the History of the Behavioral Sciences, 8, 86-102.

Laties, V. G. (1987). Society for the Experimental Analysis of Behavior: The first 30 years (1958-1987). Journal of the Experimental Analysis of Behavior, 48, 495-512.

Laties, V. G. (2008). The Journal of the Experimental Analysis of Behavior at fifty. Journal of the Experimental Analysis of Behavior, 89, 95-109. doi: 10.1901/jeab.2008.89-95

Lindsley, O. L. (1987). Collecting the first dollars for JEAB. Journal of the Experimental Analysis of Behavior, 48, 469-471.

Matos, M. A. (1996). Contingências para a análise comportamental no Brasil. Psicologia: Teoria e Pesquisa, 12, 107-111.

Marx, M. H., Henderson, R. L., \& Roberts, C. L. (1955). Positive reinforcement of the bar pressing response by a light stimulus following dark operant pretests with no after effect. Journal of Comparative and Physiological Psychology, 48,73-76.

Michael, J. (1979). Flight from behavior analysis. The Behavior Analyst, 3, 1-22.

Myers, C. (1970). Journal citations and scientific eminence in contemporary psychology. American Psychologist, 9, 10411048.

Perlman, D. (1980). Who's who in psychology: Endler et al.'s SSCI scores versus a textbook definition. American Psychologist, 35, 104-106.

Peterson, M. E. (1978). The Midwestern Association on Behavior Analysis: Past, present, future. The Behavior Analyst, 1, 3-15.

Peterson, M. E. (1979). A reply to Dinsmoor. The Behavior Analyst, 2,25 .

Poling, A., Picker, M., Grossett, D., Hall-Johnson, E., \& Holbrook, M. (1981). The schism between experimental and applied behavior analysis: Is it real and who cares? The Behavior Analyst, 4, 93-102.

Reed, D. D. (2014). Determining how, when, and whether you should publish outside the box: Sober advice for early career behavior analysts. The Behavior Analyst, 37, 83-86. doi: 10.1007/s40614-014-0012-3 
Rodrigues, M. E. (2002). Behaviorismo radical: Mitos e discordâncias. Cascavel, PR: Edunioeste.

Rucci, A. J., \& Tweney, R. D. (1980). Analysis of variance and the "second discipline" of scientific psychology. Psychological Bulletin, 87, 166-184.

Rutherford, A. (2009). Beyond the box: B. F. Skinner's technology of behavior from laboratory to life, 1950-1970s. Toronto: University of Toronto Press.

Schlinger, H. D. (2014). Publishing outside the box: Unforeseen dividends of talking to strangers. The Behavior Analyst, 37, 77-81. doi: 10.1007/s40614-014-0010-5

Schoenfeld, W. N. (1987). Reminiscences, you say? Journal of the Experimental Analysis of Behavior, 48, 464-468.

Sidman, M. (1960). Tactics of scientific research: Evaluating experimental data in psychology. New York: Basic Books

Skinner, B. F. (1979). The shaping of a behaviorist: Part two of an autobiography. New York: Alfred A. Knopf.

Skinner, B. F. (1984). A matter of consequences. New York: University Press.

Skinner, B. F. (1987). Antecedents. Journal of the Experimental Analysis of Behavior, 48, 447-493.

Smith, N. G., \& Morris, E. K. (2004). A tribute to B. F. Skinner at 100: A review and chronology of his awards and honors. European Journal of Behavior Analysis, 5, 121-128.
Todorov, J. C. (2008). Uma revisão de revisões por ocasião dos 70 anos de O Comportamento dos Organismos. Revista Brasileira de Análise do Comportamento, 4, 1-7. doi: http://dx.doi. org/10.18542/rebac.v4i1.839

Todorov, J. C. (2013). Why I am not a radical behaviorist. Behavior \& Philosophy, 41, 70-75.

Tourinho, E. Z. (1999). Estudos conceituais na análise do comportamento. Temas em Psicologia da SBP, 7, 213-222.

Ulman, J. D. (2014). The Ulman-Skinner letters. European Journal of Behavior Analysis, 15, 11-19.

Verhave, T. (1987). Faded images. Journal of the Experimental Analysis of Behavior, 48, 462-464.

Wendt, R. (1949). The development of a psychological cult. American Psychologist, 4, 426-429.

Winston, A. S., \& Blais, D. J. (1996). What counts as an experiment? A transdisciplinary analysis of textbooks, 1930-1970. American Journal of Psychology, 109, 599-616.

Woodward, W. R. (1996). Skinner and behaviorism as cultural icons: From local knowledge to reader reception. In L. D. Smith \& W. R. Woodward (Eds.), Skinner and behaviorism in American culture (pp. 7-34). Bethlehem, PA: Lehigh University Press.

Wright, G. D. (1970). A further note on ranking the important psychologists. American Psychologist, 25, 650-651.

Wyatt, W. J., Hawkins, R. P., \& Davis, P. (1986). Behaviorism: Are reports of its death exaggerated? The Behavior Analyst, 9, 101-105. 